\title{
Quality of Service Optimization of Multicast Routing Protocols in Mobile Ad-Hoc Networks: AM Route, PUMA and ODMRP
}

\author{
M. Vijayalakshmi \\ Associate Professor \\ Department of Electronics and Communications, \\ GNITS \\ Hyderabad
}

\author{
D. Sreenivasa Rao, $\mathrm{PhD}$ \\ Professor, \\ Department of Electronics and Communications, \\ JNTUH \\ Hyderabad
}

\begin{abstract}
Since the structure is completely mobile, lack of central coordination, multi hop communications and limited resources provided by MANETs, QoS techniques need to be optimize the scare resource. A lot of research has been focused on providing QoS assurance in MANETs. The most important QoS metrics are packet delivery ratio, delay, through put and energy. It is a challenging issue to incorporate QoS to ad-hoc multicasting. In this paper the QoS metrics of multicast routing protocols ODMRP, AM Route and PUMA have been simulated using NS2 and analyzed.
\end{abstract}

\section{General Terms}

This paper exposes the simulation and performance analysis of ODMRP, AM Route and PUMA over 25, 30, 35, 40,45 mobile nodes using NS2.34 simulator.

\section{Keywords}

MANETS, Multicast Routing ODMRP, AM Route, PUMA, QoS metrics

\section{INTRODUCTION}

MANETs is a collection of self organizing mobile nodes that form a temporary and dynamic wireless network on a shared wireless channel with non-fixed networking infrastructure. A communication session is achieved by either through single hop transmission if recipient is within the transmission range of the source node or by relaying through intermediate nodes. For this reason MANETs are also called multi-hop packet radio network [2],[4].Each mobile node in ad-hoc routing protocol that allows itself to discover multi -hop paths through the network to any other node.

Qos protocols play major part in a QoS mechanism, since it is their responsibility to serve an application's requirements. Most applications based on voice and video requires some level of Qos constraints such as throughput, delay, energy efficiency and packet delivery ratio etc. In this paper QoS constraints such as throughput, delay, packet delivery ratio and energy for multicast routing protocols have been implemented using NS2 for routing protocols AM Route, PUMA and ODMRP.

The paper is organized as follows: I session is Introduction, II session describes about classification of multicast routing protocols , III session gives the description of routing protocols mentioned, IV session gives QoS metrics and performance of the protocols based on this, V session contains simulation details and in VI session of the paper gives the conclusions and future scope.

\section{CLASSIFICATION OF MULTICAST ROUTING PROTOCOLS}

In view of the absence of fixed infrastructure, ad-hoc networks are characterized as infrastructure-less. There is no dissimilarity between host and router, as all nodes, including mobile hosts, are required to compute, maintain, and store routing information.

There are many benefits of multicasting in MANET's. Some of them are:

i. To deliver data to many destinations simultaneously,

ii. To deliver the messages over each link of the network only once and

iii. To create copies only when the links to the destination split etc. Routing protocols can be classified based on topology, routing mechanisms, maintenance approach, initialization etc.

The various multicasting routing protocols are grouped on the basis of the manner of building routes in a MANET group such as tree, mesh, stateless and hybrid as follows:

\subsection{Tree-Based Techniques}

This technique builds a route with grouping of a variety of multicast trees of source and receiver pair from source to receiver. For every source to destination, there is existence of only one route between them. The plus of this technique is high data forwarding effectiveness and little operating cost. The high mobility in network decreases competence and reliability in terms of delivery of packet of this technique. The absence of alternate paths results in low robustness due to unpredictable change of topology due to mobility. Source or shared tree based approach is used for creation of routing paths. In source based technique, individual shortest multicast path is formed from each sender to receiver node. The mobility in MANET amplifies the traffic overhead for this method. Single distributed tree is mutual amongst all the sender nodes in shared tree method. A rendezvous point (RP) for group is there. Senders' node drive packets to RP and various receivers join at RP. There is less delay in the source based approach, whereas traffic load is skewed in the later approach. AMRIS [3] and MAODV [16] protocols are based on this technique.

\subsection{Mesh-Based Techniques}

There exist multiple routes linking source and destination node in this technique. Accessibility of numerous routes put in robustness from varies of topology due to mobility in MANET. This method is superior than the tree based practice 
in terms of high performance, increases in delivery of packets and delays to be less. However, the maintenance of multiple routes and accessibility to them and forwarding the packet results in high cost than tree based techniques. Also, the ease of use of numerous routes shall outcome in the receiving of duplicate data packets at the destination node which domino effect in enhance of network traffic and load. On-Demand Multicast Routing Protocol(ODMRP)[10],11], Core Assisted Mesh Protocol (CAMP) [18], Forwarding Group Multicast Protocol (FGMP) [2],[19] are based on this technique.

\subsection{Stateless Multicast}

As mobility in the MANET lead to origins the overhead in preserving the route and forming novel routes due to recurrent topology alterations. There is overhead in uphold tree and mesh in tree based method and mesh based method respectively. The stateless multicast procedure diminishes this overhead by explicitly release the list of destinations in data packet header by sender node. This multicasting procedure is mainly appropriate for the small sized groups. Basic routing protocol further sends the packet to all receivers. However, in huge sized groups this method raises operating cost due to broaden in list of destination addresses. Differential Destination Multicast (DDM) protocol [17] uses this procedure.

\subsection{Hybrid Approaches}

Hybrid procedures are based on a mixture of both the tree based and the mesh based approaches, and these also come across a point out in the study on the subject matter [12]. The focus of this procedure is to detain benefit of the pros of the mesh (i.e. robustness) and tree (i.e. low overhead) based methods to attain superior recital. This planned method is added extra reliability than tree based approach and diminishes the network traffic and load. AM Route [13], MCEDAR [12] multicasting protocols uses this scheme.

\section{PORTRAYAl OF ROUTING PROTOCOLS}

\subsection{On Demand Multicasting Routing Protocol (ODMRP)}

ODMRP [1], [5],[10] is one of the reliable mesh based multicast routing mechanism. In ODMRP, group membership and multicast routes are established and updated on-demand by the source. It comprises of request and reply phase similar to on-demand unicast routing mechanisms. Whenever a multicast group member desires to send packets to other members, the request phase begins. In the request phase, the source broadcasts a packet called JOIN REQUEST periodically to the entire network that acts as member advertising packet. The periodic transmission of JOIN REQUEST refreshes the membership information and updates the route in the following steps:

i. When a node receives a non-duplicate JOIN REQUEST, it stores the upstream node ID (i.e., backward learning) and rebroadcasts the packet.

ii. When the JOIN REQUEST packet reaches a multicast destination, the destination creates or updates the source entry in its member table.

iii. While valid entries exist in the member table, JOIN TABLEs are broadcasted periodically to the neighbors.

iv. When a node receives the JOIN TABLE packet, it checks if the next node ID of one of the entries matches its own ID. If it matches, the node realizes that it is on the path to the source and thus becomes a part of the forwarding group.

Later, the node sets a flag known as the forwarding group flag and broadcasts its own JOIN TABLE which is built upon matched entries. The JOIN TABLE is thus propagated by each forwarding group member until it reaches the multicast source via shortest path. This process constructs (or updates) the routes from source to destinations and builds a mesh of nodes called as forwarding group. The nodes in forwarding group are responsible to forward multicast packets to all the group members. The nodes involved in all the forwarding groups of a network are fully connected which forms a mesh structure. Forwarding group nodes support shortest paths between any member pairs. Note that a multicast destination node can also be a forwarding group node if it is on the path between a multicast source and another destination. This type of mesh structure enables richer connectivity among multicast members.

\section{Advantages}

i. It uses soft state approach for joining/leaving of member nodes and all existing nodes update their database to keep recent routing information.

ii. Being robust in handling link and node failures, ODMRP has high packet delivery ratio and low control overheads.

iii. Another advantage is its ability to function as both unicast and multicast.

Limitations

i. Child node disconnection in case of parent node failure.

ii. Periodic invigorating of the routes to maintain connectivity during node mobility.

iii. Overhead of maintaining redundant mesh routes

iv. Exponential growth in number of control packets with increase in number of nodes due to its broadcasting nature.

v. Reduced scalability.

\subsection{Ad Hoc Multicasting Routing Protocol (AM Route)}

AM Route [2],[8] creates a multicast shared-tree over mesh with bidirectional shared multicast tree using unicast tunnels to provide connections between multicast group members. Each group has at least one logical core that is responsible for group members and tree maintenance.

Table 1: Routing mechanisms and performance metrics of ODMRP, PUMA \& AM Route

\begin{tabular}{|l|l|l|l|l|l|l|l|}
\hline Protocol & $\begin{array}{l}\text { Route } \\
\text { discovery }\end{array}$ & $\begin{array}{l}\text { Routing } \\
\text { mechanism }\end{array}$ & $\begin{array}{l}\text { Routing } \\
\text { efficiency }\end{array}$ & Reliability & $\begin{array}{l}\text { Control } \\
\text { overhead }\end{array}$ & Scalability & QoS \\
\hline ODMRP & $\begin{array}{l}\text { Source } \\
\text { initiated }\end{array}$ & Reactive & High & Medium & Medium & High & Low \\
\hline
\end{tabular}




\begin{tabular}{|l|l|l|l|l|l|l|l|}
\hline AM Route & Hybrid & Proactive & High & High & High & Low & Low \\
\hline PUMA & $\begin{array}{l}\text { Receiver } \\
\text { initiated }\end{array}$ & Reactive & High & High & Low & High & High \\
\hline
\end{tabular}

Initially, each group member declares itself as a core for its own group of size one. Each core periodically floods Join Requests (JREQs) to discover other disjoint mesh segments for the group. Any member, either core or non-core in the mesh segment, can respond to the JREQ message to avoid adding many links to a core. According to the core resolution algorithm, among all cores, one of them will be the logical core.

After the mesh has been created, the logical core periodically transmits TREECREATE control packets to mesh neighbors in order to build a multicast shared tree. When a member node receives a non-duplicate TREECREATE from one of its mesh links, it forwards the packet to all other mesh links. If a duplicate TREECREATE packet is received, a TREECREATE-NAK is sent back along with the incoming link. The node receiving a TREECREATE-NAK (TREECREATE-negative acknowledgement) marks the link as a mesh link instead of a tree link. The nodes wishing to leave the group send the JNAK (Join-Negative Acknowledgement) message to the neighbors and do not forward any data packets for the group.

Advantages

i. AM Route creates a competent and robust shared tree for each group.

ii. It helps to keep the multicast delivery tree unchanged with changes of network topology as long as paths between tree members and core nodes exist via mesh links.

Limitations

i. When the mobility is present, AM Route suffers from loop formation, creates non- optimal trees, and requires higher overhead to assign a new core.

ii. AM Route also suffers from a single point of the core node's failure.

\subsection{Protocol for Unified Multicasting through Announcement (PUMA)}

PUMA [10],[14] is used in ad hoc network. It does not require any pre-assigned core and unicast routing protocol for its operation. Very simple multicast announcement signaling is used here for the creation and maintenance of the multicast routing structure. It uses a receiver initiated approach, in which the receiver elects a core to serve as the point of contact between the group and non-members of the group. The multicast receivers connect the core through the shortest path between the core and the individual receiver. The nodes on the shortest paths between any receiver and the core collectively form the mesh structure.

Multicast announcement is a single control message used in PUMA for all its functions. This control message gives the details about sequence number, group ID, core ID, distance to the core and parent details. Parent indicates the preferred neighbor to reach the core. The core of the group transmits these multicast announcements every three seconds periodically. Whenever there is a change in the user member status, during that time also a new multicast announcement was generated. If a receiver wants to join a multicast group, then it verifies first whether it has received a multicast announcement for that group or not.

If the multicast announcement is already received then the core specified in that announcement is taken as its core. If the announcement is not received then it considers itself as a core for the group and starts to send a new announcement to its neighbor. If several receivers try to join the group at a time, than the receiver with highest ID was elected as the core for that group.

In PUMA[13], the multicast packets move hop by hop, until they reach the mesh members. A node forwards a multicast packet it receives from the neighbors if it is the parent for that neighbor's node. Once the data packets reach the mesh, they are flooded within the mesh. Packet ID cache is used to detect and discard the duplicate packets. Comparison of the protocols based on different characteristics is given in Table 1.

Advantages

i. It provides high robustness,

ii. It also has more adequate channel access.

Limitations

i. Intrusion will become major problem in presence of more no of groups.

\section{QoS METRICS AND PERFORMANCE EVALUATION OF PROTOCOLS}

We have used packet delivery ratio, throughput, end-to-end delay and average energy of multicast routing protocols (PUMA, AM Route and ODMRP) to evaluate the QoS.

\subsection{Packet Delivery Ratio}

It is defined as the ratio of number of data packets delivered to all the receivers to the number of data packets supposed to be delivered to the receivers. This ratio represents the routing effectiveness of the protocol:

PDR = Packets delivered to the destination/Packets sent by the source

Table: Packet Delivery Ratio (PDR)

\begin{tabular}{|l|l|l|l|}
\hline Nodes & AM Route & PUMA & ODMRP \\
\hline 25 & 74.95 & 75.96 & 1.39 \\
\hline 30 & 86.99 & 88.36 & 1.5 \\
\hline 35 & 93.97 & 99.02 & 1.76 \\
\hline 40 & 106.44 & 112.23 & 1.32 \\
\hline 45 & 126.53 & 105.92 & 1.22 \\
\hline
\end{tabular}




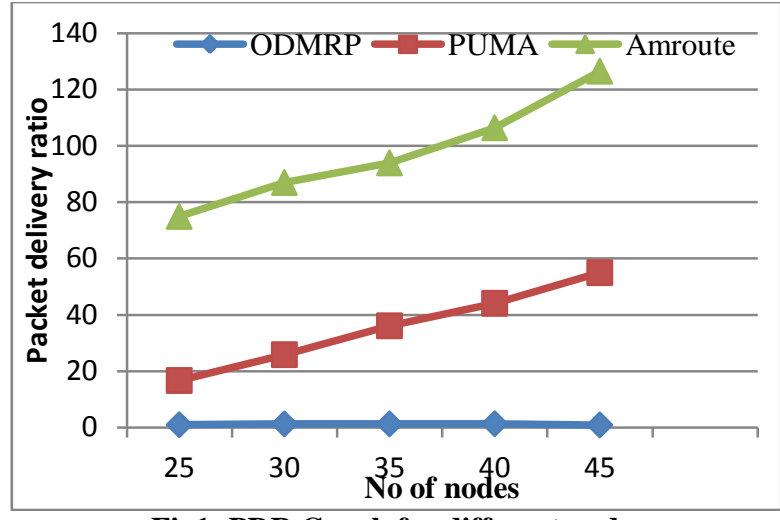

Fig1: PDR Graph for different nodes

\subsection{Average End-to-End Delay}

It is the average time taken for a data packet to move from the source to the receivers:

Avg. EED $(\mathbf{m s})=$ Total EED $/$ No. of packets sent

Table: End to End Delay (ms)

\begin{tabular}{|c|c|c|c|}
\hline Nodes & AM Route & PUMA & ODMRP \\
\hline 25 & 0.177 & 0.147 & 0.961 \\
\hline 30 & 0.185 & 0.184 & 0.934 \\
\hline 35 & 0.181 & 0.191 & 0.964 \\
\hline 40 & 0.204 & 0.181 & 1.171 \\
\hline 45 & 0.177 & 0.164 & 1.143 \\
\hline
\end{tabular}

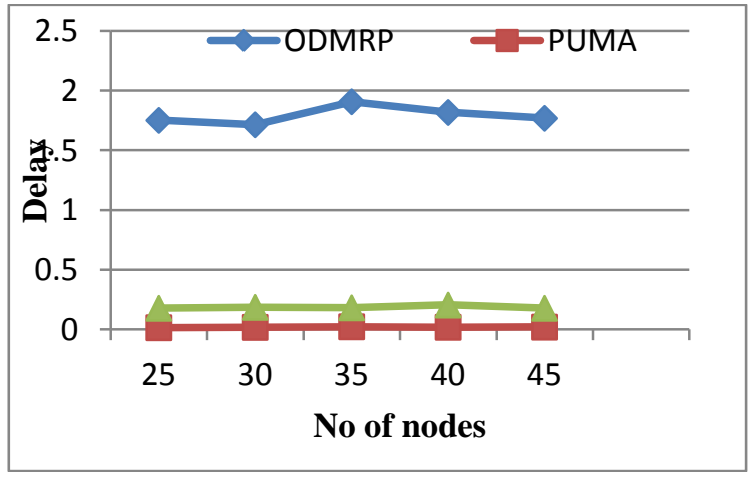

Fig2: Delay Graph for different nodes

\subsection{Throughput}

Throughput refers to how much data can be transferred from the source to the receiver(s) in a given amount of time:

Throughput $($ Kbps $)=$ Number of packets sent $/$ Time Taken (3)

Table : Throughput(Kbps)

\begin{tabular}{|l|l|l|l|}
\hline Nodes & AM Route & PUMA & ODMRP \\
\hline 25 & 3175.91 & 3296.46 & 56.49 \\
\hline 30 & 3889.01 & 3862.97 & 59.83 \\
\hline 35 & 4231.71 & 4262.24 & 92.48 \\
\hline 40 & 4649.61 & 5019.63 & 54.74 \\
\hline 45 & 5486.64 & 4661.86 & 66.72 \\
\hline
\end{tabular}

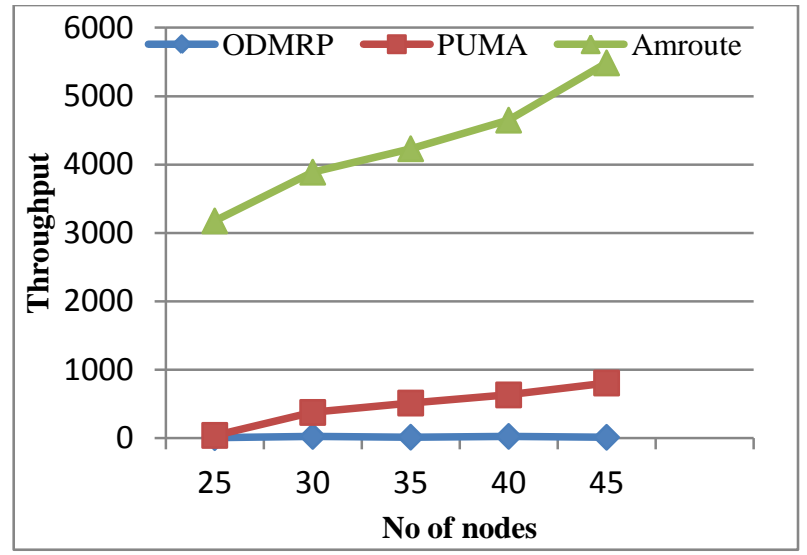

Fig3: Throughput Graph for different Nodes

\subsection{Average Energy}

Energy consumption of the network is the sum of energy consumption of all the nodes in the network. Energy consumption each node is the difference between the energy of the node at the start of the communication and the energy of the node at the end of the communication.

Energy consumption (Joules) $=\sum$ (Initial energy - Residual Energy)

Table: Average Energy

\begin{tabular}{|c|c|c|c|}
\hline Nodes & AM Route & PUMA & ODMRP \\
\hline 25 & 85.1442 & 85.1444 & 93.6 \\
\hline 30 & 101.454 & 101.458 & 111.6 \\
\hline 35 & 117.728 & 117.707 & 129.6 \\
\hline 40 & 134.047 & 134.07 & 147.6 \\
\hline 45 & 150.377 & 150.283 & 165.59 \\
\hline
\end{tabular}

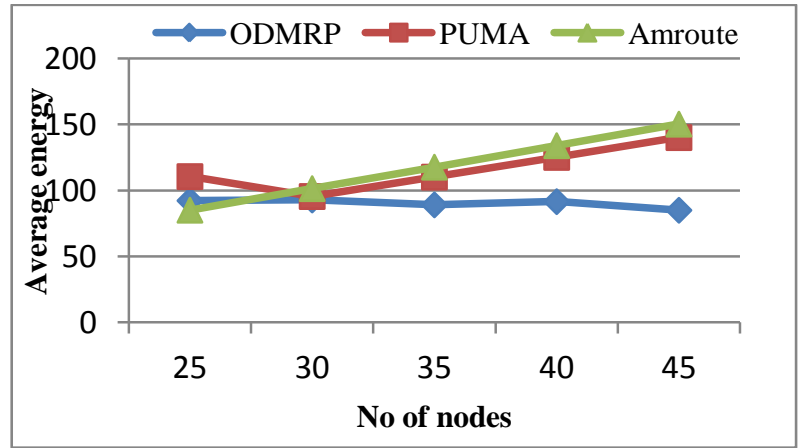

Fig4: Average Energy Graph for different Nodes

\section{SIMULATION MODEL}

Simulation of protocols is carried out by using NS2.The simulation scenario is given in Table 3 below.

After the QoS metrics evaluation of routing protocols (ODMRP, PUMA and AM Route) comparisons are framed in table2.PUMA is more suitable for video steaming applications. In ODMRP Packet delivery ratio decreases with increase in number of senders due to congestion. 
Table2: Performance comparisons of the multicast routing protocols based on QoS metrics

\begin{tabular}{|l|l|l|l|l|}
\hline Protocols & PDR & Throughput & Delay & $\begin{array}{l}\text { Avg. } \\
\text { Energy }\end{array}$ \\
\hline ODMRP & Less & Less & More & Less \\
\hline PUMA & More & More & Less & More \\
\hline $\begin{array}{l}\text { AM } \\
\text { Route }\end{array}$ & More & More & Less & More \\
\hline
\end{tabular}

Table3: Simulation Scenario

\begin{tabular}{|l|l|}
\hline Simulator & $\begin{array}{l}\text { Network Simulator } \\
\text { (NS-2.34) }\end{array}$ \\
\hline Total Nodes & $25,30,35,40,45$ \\
\hline Simulation Time & $200 \mathrm{sec}$ \\
\hline Simulation Area & $1000 \times 1000$ \\
\hline Propagation Model & Two Ray Ground Model \\
\hline Pause Time & $0-10$ sec \\
\hline MAC Protocol & MAC_802.11 \\
\hline Data Packet Size & 512 bytes \\
\hline Data Rate & 11 Mbps \\
\hline Antenna & Omni Directional Antenna \\
\hline IFQ Length & 50 packets \\
\hline Bandwidth of Physical Layer & 11 Mbps \\
\hline Routing Protocol & ODMRP, AMRoute \& \\
\hline Mobility Speed & $0-10$ m/sec \\
\hline Traffic & CBR \\
\hline
\end{tabular}

\section{CONCLUSIONS}

AM Route is routing protocol compared with PUMA and ODMRP. Throughput, Energy and PDR are high compared with other protocols. For this scenario PUMA also performs as good as this protocol. In PUMA, the number of packets send is high and the number of total packets transmitted per data packets delivered is lower than the ODMRP. It means the channel access of PUMA is more efficient than ODMRP. It is also found that, PUMA maintains almost constant EED with multiple sender scenarios also. This makes, PUMA as a more suitable protocol for video streaming applications.

\section{REFERENCES}

[1] M. Gerla, S. -J Lee, and W. Su. "On-Demand Multicast Routing Protocol (ODMRP) for Ad Hoc Networks," Internet draft, draft-ietfmanet-odmrp-02.txt, 2000

[2] C. Siva Ram Murthy and B. S. Manoj, "Ad Hoc Wireless Networks, Architectures and Protocols", Second Edition, Low price Edition, Pearson Education, 2007.

[3] J. J. Garcia-Luna-Aceves and E. L. Madruga, "The Core Assisted Mesh Protocol." IEEE JSAC, Aug. 1999, pp 1380-94

[4] S. Corson and J. Macker. - Mobile ad hoc networking (MANET): Routing protocol performance issues and evaluation considerations\|, RFC 2501, January 1999, available at http://www.ietf.org/rfc/rfc2501.txt.

[5] Thomas Kunz and Ed Cheng, - Multicasting in Ad-Hoc Networks: Comparing MAODV and ODMRP\|, Carleton University, Proceedings of the workshop on Ad hoc Communications, 2001.

[6] T. Kunz, "Multicasting: From fixed networks to ad-hoc networks",Handbook of Wireless Networks and Mobile Computing, John Wiley \& Sons, 28 Feb 2002.

[7] Chae Young Lee and Hee Kwun Cho, -Multicast Routing Considering Reliability and Network Load In Wireless Ad-Hoc Network\| IEEE, VETECS, 2001.

[8] E. Bommaiah, M. Liu, A. McAuley, and R. Talpade, AMRoute:Ad-hoc Multicast Routing Protocol, Internetdraft, draft-talpade-manetamroute-OO.txt, Aug. 1998 ,

[9] Upkar Varshney, -Multicast over Wireless Networksll, communications of the ACM, Dec 2002, vol45, no 12

[10] J. Rangarajan, K.Bhaskaran,” Performance Analysis Of Multicast Protocols: ODMRP, PUMA AND OBAMP”,IJCSC,vol2,no.2, July-December 2011,pp.577581

[11] Su W Lee SJ, Gerla M.," On Demand Multicast Routing Protocol in Multihop Wireless Mobile Networks(ODMRP)," Springer Link Mobile Networks and Applications 2002;7,p.441-453

[12] Rajashekhar C.Biradar, Sunilkumar S.Manvi, "Review Of Multicast Routing Mechanisms In Mobile Ad hoc Networks," ELSEVIER Journal of Network and Computer Applications, 2012, vol 35, pp.221-239 doi:10.1016/j.jnca.2011.08.003

[13] Mandeep Singh, Prakash Rao Ragiri," Multicast Routing Protocol (Puma) Security Issues In Mobile Ad Hoc Networks,"IJTRE, Volume 2, Issue 11, July-2015 ISSN (Online): 2347 - 4718,p:2752-2757.

[14] Xiaozhou Li, Shiv Muthukumar, PUMA: policy-based unified management architecture for wireless networking. , 2010

[15] Luo Junhai, Ye Danxia, Xue Liu, and Fan Mingyu ,"A Survey of Multicast Routing Protocols for Mobile AdHoc Networks", IEEE Communications Surveys \& Tutorials, Vol. 11, No. 1, First Quarter 2009,pp:78-91.

[16] EM Royer ,Perkins CE, "Multicast Operation of the Ad hoc on Demand Distance Vector Routing Protocol (MAODV).IEEE conference on mobile computing and networking,WMCSA,Seattle,Febraury,1999,p.207-218

[17] Lusheng Ji and M. Scott Corson. Differential destination multicast-a manet multicast routing protocol for small groups. In INFOCOM 2001, volume 2, pages 1192 1201, Anchorage, AK USA, Apr. 2001.

[18] J.J. Garcia-Luna-Aceves and E. Madruga, "The Core Assisted Mesh Protocol", accepted for publication in IEEE Journal on Selected Areas in Communications, Special issue on Ad-Hoc Networks, 1999.

[19] Ching-Chuan Chiang, Mario Gerla, Lixia Zhang, "Forwarding group multicast protocol (FGMP) for multihop", mobile wireless networks, Cluster Computing 1, (2), 1998 pp:187-196. 